\title{
Histone modifications during tobacco male meiosis
}

\author{
S. MURSALIMOV ${ }^{1}$, E. DEINEKO ${ }^{1}$, A. HOUBEN ${ }^{2}$, and D. DEMIDOV ${ }^{2 *}$ \\ Institute of Cytology and Genetics, Russian Academy of Sciences, Siberian Branch, \\ Novosibirsk 630090, Russia ${ }^{1}$ \\ Leibniz Institute of Plant Genetics and Crop Plant Research, Gatersleben 06466, Germany²
}

\begin{abstract}
The special and temporal distribution of several posttranslational histone modifications (H3K4me2, H3K4me3, H3K9me2, H3K27me2, H3K9ac, H3K14ac, H3K18ac, H3K27ac, H3S10ph, H3S28ph, H3T3ph, H3K4me1-H3T3ph, H3K4me3/ H3T3ph H3T11 ph, H2AT121 ph, and H2A.XS139ph) has been analysed during microsporogenesis of tobacco. Homogeneous distribution of the euchromatin marks $\mathrm{H} 3 \mathrm{~K} 4 \mathrm{me} 2$ and $\mathrm{H} 3 \mathrm{~K} 4 \mathrm{me} 3$ throughout the entire chromatin of amphidiploid Nicotiana tabacum suggests that both subgenomes were transcriptionally active. Localization of hyperacetylated clusters of H3K18 and H3K27 close to nucleoli suggests that both modifications were possibly involved in transcription of the 45S rDNA. A subset of chromosomes showed clusters of hypermethylated H3K9me2 and H3K27me2 regions. The cell-cycle dependent phosphorylation of histone $\mathrm{H} 3$ during meiosis revealed a high conservation compared to other plant species. In addition to meiotic prophase I, we observed phospho-H2A.X signals during the second stage of meiosis in N. tabacum.
\end{abstract}

Additional key words: chromatin marks, microsporogenesis, Nicotiana tabacum.

\section{Introduction}

Histones are the major protein components of the chromatin, being responsible for its structure and functional state. Several types of posttranslational histone modifications are known, in particular, acetylation, phosphorylation, methylation, ubiquitination, ADP-ribosylation, and SUMOylation (Kouzarides 2007). These changes are associated with chromosome condensation/segregation, transcription activation/inhibition, recombination, DNA repair, and apoptosis (Fuchs and Schubert 2012).

The chromosomal distribution of histone modifications have been studied in several plant species including wheat, rye, maize, Arabidopsis thaliana, and Vicia faba (Kaszas and Cande 2000, Gernand et al. 2003, Houben et al. 2005, Carchilan et al. 2007). Despite that the core histones as well as their posttranslational modification are highly conserved, the distribution of individual modification types along chromosomes may differ in different plant species as well as in individual cell types (Fuchs et al. 2006, Houben et al. 2013).

Phosphorylation of histones during meiosis is important for normal chromosome condensation and segregation, but only in a limited number of species, it has been studied in detail (Houben et al. 2007, Sharma et al. 2015). Acetylation and methylation of histones were studied in plants more intensively (Fuchs et al. 2006, Fuchs and Schubert 2012), but rarely in meiotic tissues.

Nicotiana tabacum is a model species in many areas of genetics and cytology. In contrast to A. thaliana, the main model in plant biology, N. tabacum is amphidiploid. It is likely derived from a hybridization event between N. sylvestris and N. tomentosiformis (Goodspeed 1956, Ren and Timko 2001, Murad et al. 2002, Lim et al. 2004). This characteristics of tobacco can be used in studying the epigenetic status of different parental genomes in hybrids. Moreover, tobacco is one of the few crop species with a well-established transformation procedure which routinely can be applied in many laboratories (An 1985). It is also suitable as a cytological model due to the larger chromosomes compared to A. thaliana. However up to now, no cytological analysis of the chromatin composition was performed.

In this work, we examined the distribution of major posttranslational histone modifications (methylation, acetylation, and phosphorylation) in the tobacco microsporocytes.

Submitted 15 December 2018, last revision 17 May 2019, accepted 3 June 2019.

Abbreviations: BSA - bovine serum albumin; NOR - nucleolus organiser region; PBS - phosphate buffered saline.

Acknowledgements: The work was supported by the Russian Foundation for Basic Research [16-34-60007 mol_a_dk], the Siberian Branch of the Russian Academy of Science under the program Molecular genetic bases of regulation of genes expression, morphology, differentiation and cell reprogramming [0324-2019-0042], and the German Federal Ministry of Education and Research (Plant 2030, Project 031B0192NN, HaploTools)

* Corresponding author; e-mail: demidov@ipk-gatersleben.de 


\section{Materials and methods}

Plants: For our cytological work, we selected tobacco (Nicotiana tabacum L.) cv. Petit Havana SR1 because it has a normal meiosis without any noticeable irregularities and a high pollen fertility. Five plants were grown in a greenhouse with a 16-h photoperiod, natural irradiance, day/night temperatures of $22 / 18^{\circ} \mathrm{C}$, and a relative humidity of $55 \%$.

Immunostaining squash preparations: Anthers were fixed with freshly prepared $4 \%(\mathrm{~m} / \mathrm{v})$ paraformaldehyde in $1 \times$ phosphate buffered saline (PBS) for $15-30 \mathrm{~min}$ on ice. Anthers were then washed in $1 \times$ PBS three times for $15 \mathrm{~min}$ on ice, squashed into a suspension in $0.1 \mathrm{~cm}^{3}$ of $1 \times$ PBS in an embryo dish with a blunt end of a dissection needle. Then, the meiocyte suspension was transferred to a $1.5 \mathrm{~cm}^{3}$ tube containing $0.2 \mathrm{~cm}^{3}$ of enzyme mixture containing $1 \%(\mathrm{~m} / \mathrm{v})$ pectolyase (Sigma, St. Louis, USA), $1 \%(\mathrm{~m} / \mathrm{v})$ cytohelicase (Sigma), $0.7 \%(\mathrm{~m} / \mathrm{v})$ cellulase R-10 (Duchefa, Haarlem, Netherlands), and $0.7 \%(\mathrm{~m} / \mathrm{v})$ cellulase (Merck, Darmstadt, Germany) dissolved in $1 \times$ PBS and placed to a shaking incubator at $300 \mathrm{rpm}$ and 37 ${ }^{\circ} \mathrm{C}$ for $30 \mathrm{~min}$ to digest the cell wall. Cells were centrifuged at $7000 \mathrm{~g}$ and $4{ }^{\circ} \mathrm{C}$ for $6 \mathrm{~min}$, resuspended with PBS and centrifuged again at $7000 \mathrm{~g}$ and $4^{\circ} \mathrm{C}$ for $6 \mathrm{~min}$. Then, cells were resuspended in $0.02 \mathrm{~cm}^{3}$ of $1 \times$ PBS, transferred to a poly-L-lysine-coated slide and squashed between a glass slide and cover slip. After freezing in liquid nitrogen, the cover slips were removed and the slides were transferred immediately into $1 \times$ PBS.

To avoid non-specific antibody binding, slides were incubated in $1 \times$ PBS with $3 \%(\mathrm{~m} / \mathrm{v})$ bovine serum albumin (BSA), and $0.1 \%(\mathrm{~m} / \mathrm{v})$ Tween 20 at $37^{\circ} \mathrm{C}$ in a pre-warmed moisture chamber for $15 \mathrm{~min}$, then primary antibodies were applied to the slides. The primary antibodies used were anti-H3S10ph (Abcam, Cambridge, UK, cat. No. 14955); anti-H3K4me3 (Merck, cat. No. 07473); anti-H3K9me2 (Active Motif, La Hulpe, Belgium, cat. No. 39239); anti-H3K27me2 (Merck, cat. No. 07452); anti-H3K4me2 (Merck, cat. No. ABE250); antiH3T11ph (Merck, cat. No. 07-492); anti-H2A.XS139ph (Cell Signaling, Leiden, Netherlands, cat. No. 2577); anti-H3K9ac (Thermo Scientific, Waltham, USA, cat. No. MA5-11195); anti-H3K27ac (Abcam, cat. No. 45173); anti-H3K14ac (Abcam, cat. No. 52946); anti-H3K18ac (Abcam, cat. No. 1191); anti-H2AT121ph (MyBioSource, San Diego, USA, cat. No. 004447); anti-H3T3ph (Merck, cat. No. 07-424); anti-H3K4me1-H3T3ph (Merck, cat. No. 07-554); anti-H3K4me3/H3T3ph (Abcam, cat. No. 3738); anti-H3S28ph (Merck, cat. No. H9908). All primary antibodies were diluted at 1:200 in $1 \times$ PBS, $1 \%$ BSA, and $0.1 \%$ Tween 20 .

After overnight incubation at $4{ }^{\circ} \mathrm{C}$ in a moisture chamber and washing three times for $15 \mathrm{~min}$ in $1 \times \mathrm{PBS}$ with $0.1 \%$ Tween 20 , the slides were incubated at $37^{\circ} \mathrm{C}$ for $1 \mathrm{~h}$ in a pre-warmed moisture chamber with Alexa Fluor 594- and DyLight 488-conjugated anti-rabbit IgG (Jackson ImmunoResearch, West Grove USA, cat. No. 711585-152 and 111-485-144); Alexa Fluor 488-conjugated anti-mouse IgG (Molecular probe, Eugene, USA, cat. No. A-11001); FITC-conjugated anti-rat IgG (Dianova, Hamburg, Germany, cat. No 112-095-003), diluted at 1:250 in $1 \times$ PBS, $1 \%$ BSA, $0.1 \%$ Tween 20 . Then, the slides were washed in $1 \times$ PBS with $0.1 \%$ Tween 20 three times for 15 min and mounted in ProLong Gold antifade (Life Technologies, Carlsbad, USA) containing 4',6-diamidino2-phenylindole (DAPI).
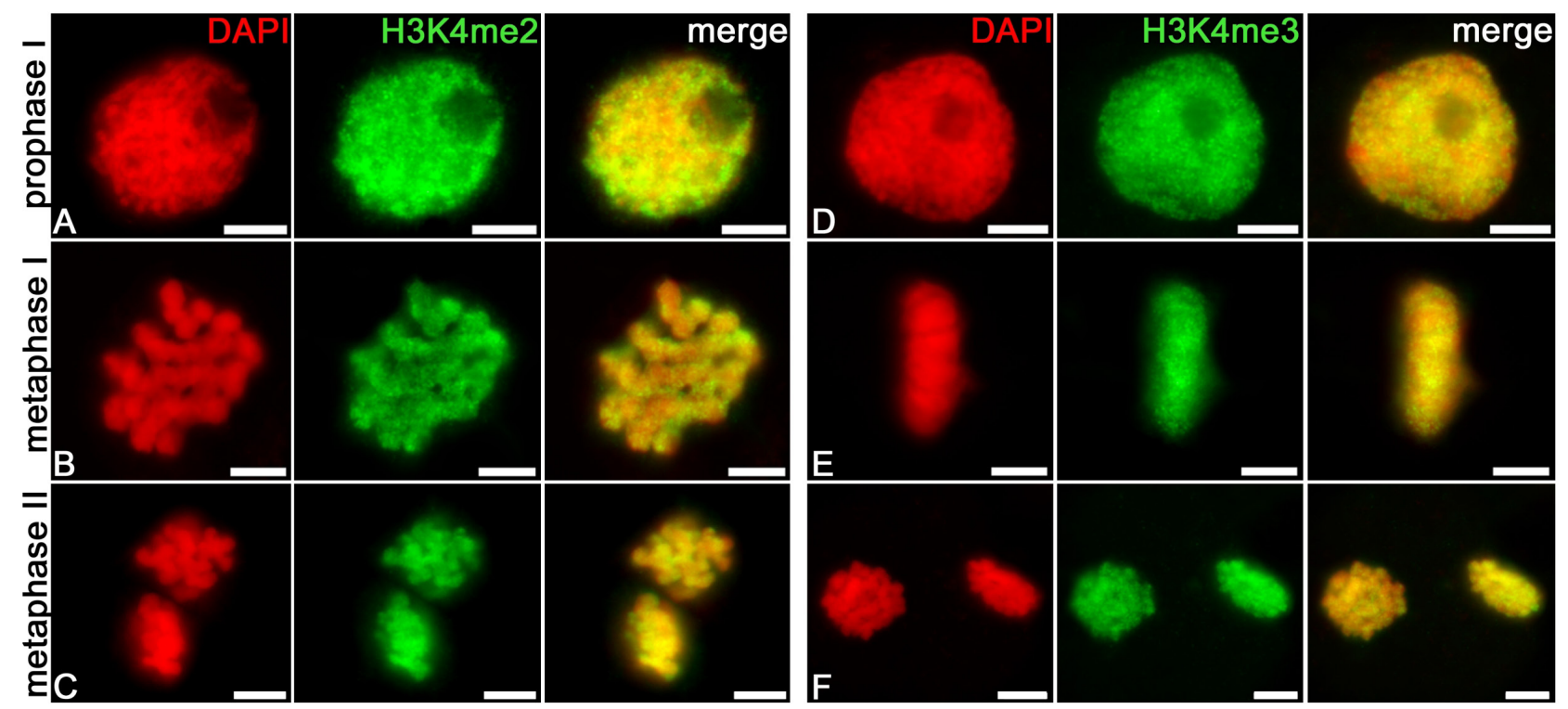

Fig. 1. The distribution of dimethylated and trimethylated histone $\mathrm{H} 3 \mathrm{~K} 4$ during microsporogenesis of tobacco: $A$ - $\mathrm{H} 3 \mathrm{~K} 4 \mathrm{me} 2$ and $D$ $\mathrm{H} 3 \mathrm{~K} 4 \mathrm{me} 3$ in prophase I (zygotene-pachytene); $B-\mathrm{H} 3 \mathrm{~K} 4 \mathrm{me} 2$ and $E-\mathrm{H} 3 \mathrm{~K} 4 \mathrm{me} 3$ in metaphase I; $C$ - H3K4me2 and $F-\mathrm{H} 3 \mathrm{~K} 4 \mathrm{me} 3$ in metaphase II. The nucleus in Fig. $1 E$ is a polar view of metaphase I. Bars are $5 \mu \mathrm{m}$. 

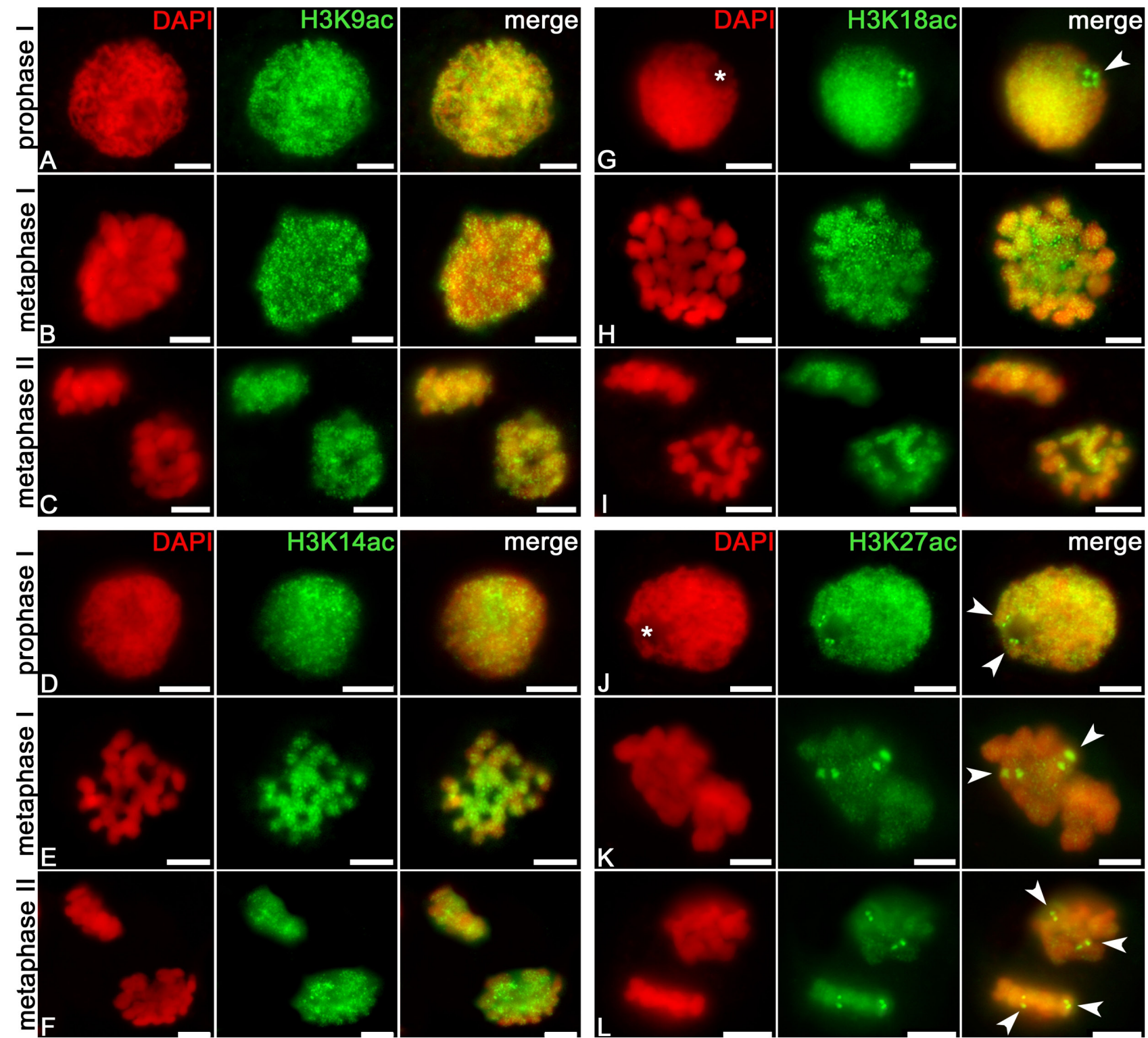

Fig. 2. The distribution of acetylated histones $\mathrm{H} 3 \mathrm{~K} 9,14,18,27$ during microsporogenesis of tobacco: $A-\mathrm{H} 3 \mathrm{~K} 9 \mathrm{ac}, D-\mathrm{H} 3 \mathrm{~K} 14 \mathrm{ac}, G$ -H3K18ac, and $J$ - H3K27ac in prophase I (zygotene-pachytene); $B-\mathrm{H} 3 \mathrm{~K} 9 \mathrm{ac}, E-\mathrm{H} 3 \mathrm{~K} 14 \mathrm{ac}, H-\mathrm{H} 3 \mathrm{~K} 18 \mathrm{ac}$, and $K$ - H3K27ac in metaphase I; $C$ - H3K9ac, $F-\mathrm{H} 3 \mathrm{~K} 14 \mathrm{ac}, I-\mathrm{H} 3 \mathrm{~K} 18 \mathrm{ac}$, and $L-\mathrm{H} 3 \mathrm{~K} 27 \mathrm{ac}$ in metaphase II. Asterisks denote nuclei and arrowheads hyperacetylated regions. Bars are $5 \mu \mathrm{m}$.

Indirect immunofluorescence: Fluorescence signals were observed using an epifluorescence microscope Olympus $B X-61$ (Hamburg, Germany). Images were captured with an ORCA-ER CCD camera (Hamamatsu, Herrsching am Ammersee, Germany), analysed, and processed with the Adobe Photoshop CS4 software. At least four slides and 200 cells were analysed for each meiotic stage and each antibody.

\section{Results and discussion}

The following posttranslational histone modifications were selected as marks to visualize transcriptionally potent chromatin during microsporogenesis of tobacco: histone H3K4me2, H3K4me3, H3K9ac, H3K14ac, H3K18ac, and $\mathrm{H} 3 \mathrm{~K} 27 \mathrm{ac}$. Immunostaining with antibodies against di- or tri-methylated lysine 4 of histone $\mathrm{H} 3$ resulted in intensive immunosignals uniformly distributed over the nuclei at the zygotene-pachytene stage (Fig. 1A,D). The signal distribution patterns observed at the first and second meiotic metaphases are similar (Fig. $1 B, C, E, F$ ). It seems that in amphidiploid tobacco, both genomes are transcriptionally potent also in the pericentromeric area. This suggests that in tobacco a high homogeneous euchromatinisation at the interphase (Fig. 1 Suppl.) and meiosis occurred. 

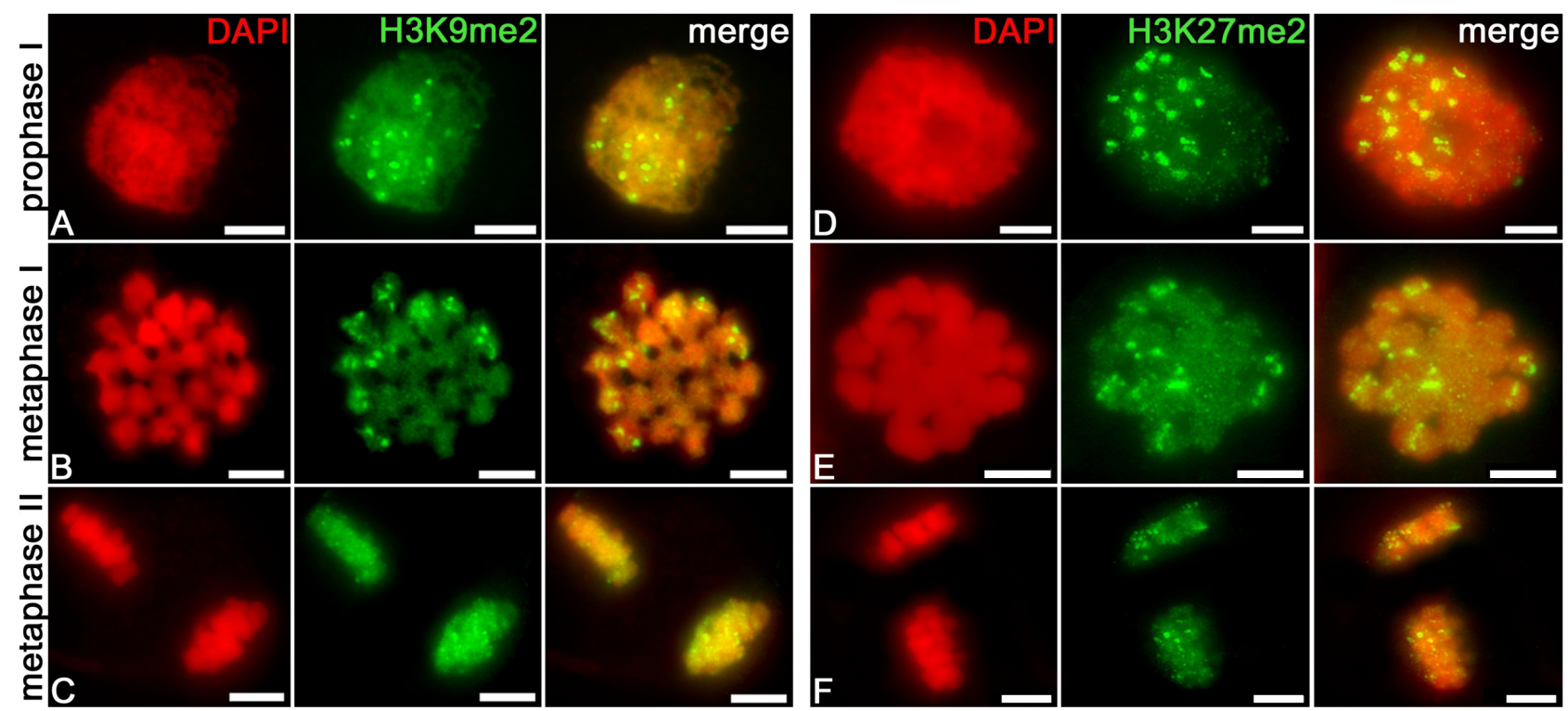

Fig. 3. The distribution of dimethylated histones $\mathrm{H} 3 \mathrm{~K} 9,27$ during microsporogenesis of tobacco: $A$ - H3K9me 2 and $D-\mathrm{H} 3 \mathrm{~K} 27 \mathrm{me} 2$ in prophase I (zygotene-pachytene); $B$ - H3K9me2 and $E$ - H3K27me2 in metaphase I; $C$ - H3K9me2 and $F$ - H3K27me2 in metaphase II. Bars are $5 \mu \mathrm{m}$.

Analysis of the distribution of histone $\mathrm{H} 3$ acetylated at lysines 9, 14, 18, and 27 demonstrated an almost uniform distribution of immunosignals in the first and second meiotic division (Fig. 2). Hyperacetylated regions were observed for the acetylated lysines 18 and 27 (Fig. $2 G, J-L$ ), which, in early prophase I, are associated with the nucleolus (Fig. $2 G, J$ ). Hyperacetylation signals from lysine 18 are detectable only at prophase I (Fig. $2 G)$ and poorly detectable at subsequent meiotic stages (Fig. $2 H, I$ ). On the other hand, the four strong H3K27ac signals were retained during all meiotic stages (Fig. $2 J-L$ ). It is interesting to note that hyperacetylated lysine 18 and 27 regions were also observed in the interphases of somatic cells (Fig. 1 Suppl.). Meiotic cells demonstrated four hyperacetylated signals as well. Most likely in the case of $\mathrm{H} 3 \mathrm{~K} 27 \mathrm{ac}$, the signals are observed in prophase I in pairs since they are double signals of bivalents. We assume that these hyperacetylation regions were likely nucleolus organiser regions (NORs), which are normally part of chromosomes S10 - 12 and T3 (Lim et al. 2004, Shibata et al. 2013).

The acetylation of histone $\mathrm{H} 3$ at $\mathrm{K} 9$ and $\mathrm{K} 14$ in tobacco meiosis was very close to Arabidopsis and differ from the immunosignal patterns observed in meiotic cells of monocots. In tobacco, the signals were distributed uniformly in the first and second meiotic divisions. In Arabidopsis, the signals are also distributed uniformly and constantly throughout meiosis, but the pericentromeric heterochromatin regions are largely devoid of signals (Oliver et al. 2013). In monocots, Aegilops and Secale, the acetylation of $\mathrm{H} 3 \mathrm{~K} 9 / \mathrm{K} 14$ is observed only in the early stages of prophases I and II and is undetectable on the condensed chromosomes (Oliver et al. 2013). Tobacco did not form any obvious $\mathrm{H} 3 \mathrm{~K} 9 \mathrm{ac}$ or H3K14ac hyperacetylated clusters.
From another side, hyperacetylated H3K9 and K14 regions which colocalise with NOR regions are found in mitotic cells of $V$. faba (Belyaev et al. 1998, Fuchs and Schubert 2012). Additionally, mitotic chromosome regions of $V$. faba and barley associated with NOR demonstrate a strong acetylation of H4K5 (Houben et al. 1996, Jasencakova et al. 2001, Fuchs and Schubert 2012). In most cases, the authors associate the formation of hyperacetylated regions with the activation of transcription of acetylated histones for the synthesis of ribosomal RNA (Fuchs and Schubert 2012).

Antibodies recognising dimethylated histones $\mathrm{H} 3 \mathrm{~K} 9$ and H3K27 are used as typical heterochromatin marks (Nakayama et al. 2001, Rougeulle et al. 2004). Prophase I nuclei displayed uniform labelling with additional regions displaying hypermethylated regions likely corresponding to regions enriched in heterochromatin (Fig. $3 A, D$ ).

At metaphase I, hypermethylated regions were present only in some chromosomes (Fig. $3 B, E$ ). In the second meiotic division, hypermethylated regions were hardly detectable likely due to a high degree of chromosome condensation (Fig. 3C,F)

Labelling the meiotic cells of tobacco with H3K27me2 and $\mathrm{H} 3 \mathrm{~K} 9 \mathrm{me} 2$ antibodies also demonstrated an enrichment of signals at subtelomeric regions (Figs. $3 B, E$ and $4 A, D$ ). It might be related to the unequal distribution of coding genes similarly as in barley (Aliyeva-Schnorr et al. 2015). Transcription inactivation of gene-enriched subtelomeric regions might be regulated via methylation of $\mathrm{H} 3$ at $\mathrm{K} 9$ and $\mathrm{K} 27$.

The distribution of hypermethylated regions was far from being random, and they were located in subtelomeric regions of about a half of the chromosomes (Fig. 3A-E and $4 A, D$ ). There is highly probable that these chromosomes belong to the $\mathrm{S}$ tobacco genome where 

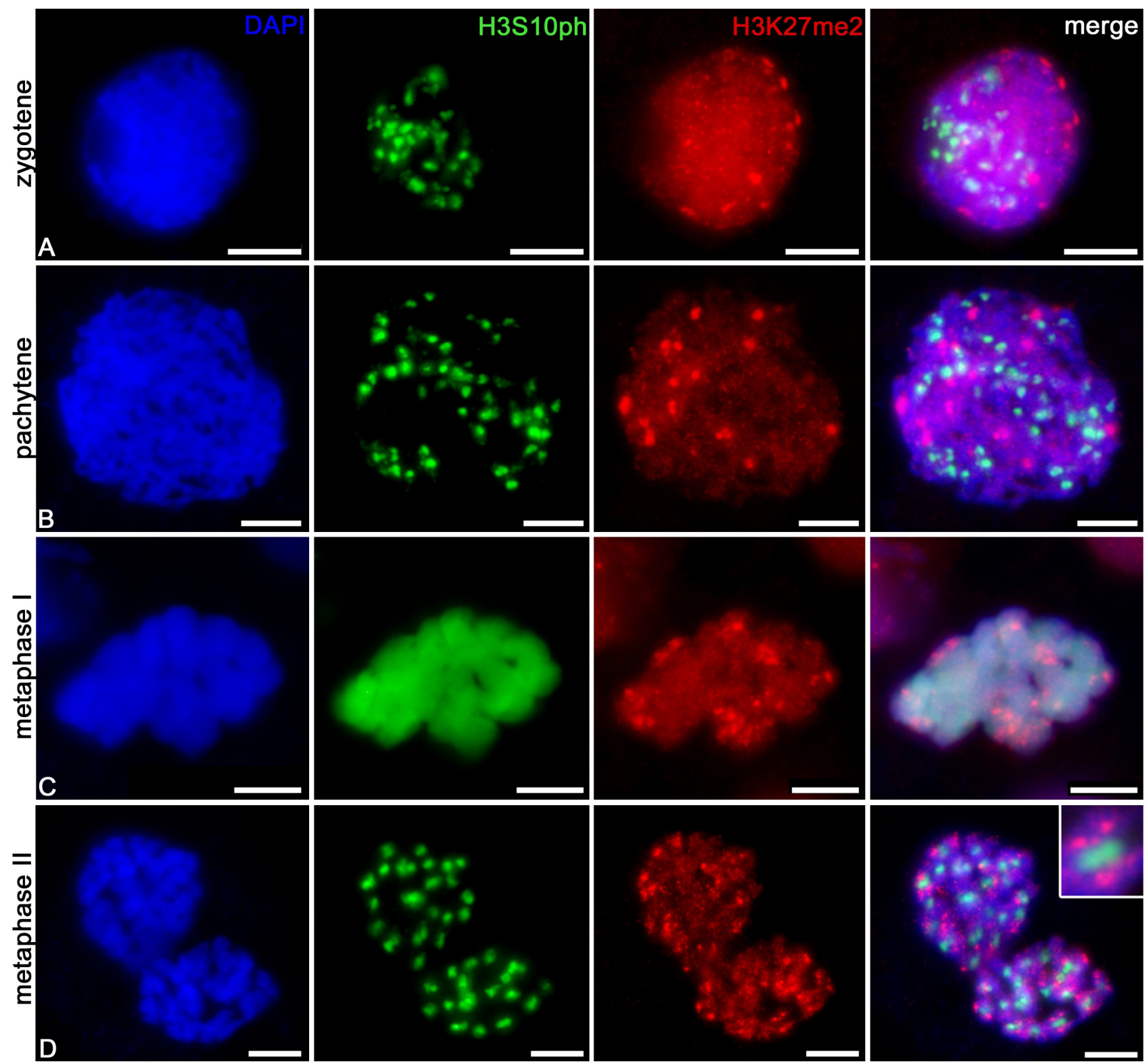

Fig. 4. The localisation of histones H3S10ph and H3K27me2 during microsporogenesis of tobacco: $A$ - zygotene, $B$ - pachytene, $C$ metaphase I, and $D$ - metaphase II. Bars are $5 \mu \mathrm{m}$.

subtelomeric satellite repeats HRS60/SYL2, forming about $2 \%$ of the tobacco genome, are located (Kenton et al. 1993, Koukalova et al. 2010). Subtelomeres of most eukaryotes are fast-evolving parts of chromosomes, and they are essential for genome stability and regular chromosome replication. An enrichment of subtelomeric regions with satellite repeats can be found in genomes of different plant species (Vershinin et al. 1995, Salina et al. 2009). In this context, it is important to note that in tobacco genome, subtelomeric repeats from $N$. sylvestris are neither lost nor introduced in the $\mathrm{T}$ genome except of several $\mathrm{S}$ to T translocations (Kenton et al. 1993, Parokonny and
Kenton 1995, Lim et al. 2000).

The phosphorylation of histones $\mathrm{H} 3$ and $\mathrm{H} 2 \mathrm{~A}$ is involved in the process of chromosome condensation and segregation (Manzanero et al. 2000, Houben et al. 2005, 2007, Kawashima et al. 2010, Dong and Han 2012). We studied the chromosomal distribution of H3S10ph, H3S28ph, H3T3ph, H3T11ph, H2AT121ph, and H2A. XS139ph. In addition, we examined the phosphorylation of threonine combined with lysine methylation, namely the variants $\mathrm{H} 3 \mathrm{~K} 4 \mathrm{me} 1 / \mathrm{H} 3 \mathrm{~T} 3 \mathrm{ph}$ and $\mathrm{H} 3 \mathrm{~K} 4 \mathrm{me} 3 / \mathrm{H} 3 \mathrm{~T} 3 \mathrm{ph}$ in tobacco microsporocytes.

The phosphorylation of histone $\mathrm{H} 3$ at serines 10 and 

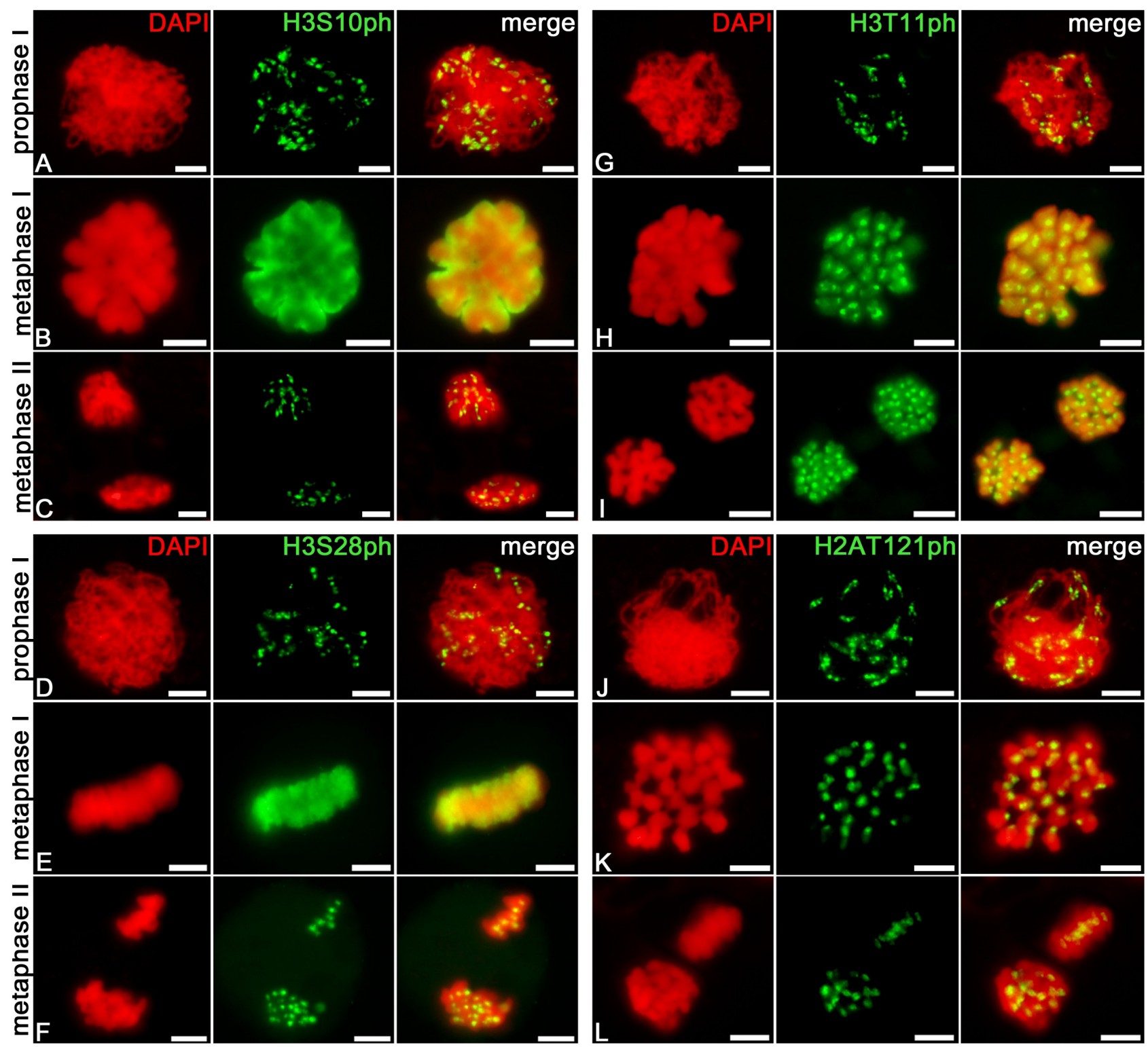

Fig. 5. The distribution of phosphorylated histones H3S10, S28, T11, and H2AT121 during microsporogenesis of tobacco: $A$ - H3S10ph, $D$ - H3S28ph, $G$ - H3T11ph, and $J$ - H2AT121ph in prophase I (zygotene-pachytene); $B$ - H3S10ph, $E$ - H3S28ph, $H$ - H3T11ph, and $K$ - H2AT121ph in metaphase I; $C$ - H3S10ph, $F$ - H3S28ph, $I$ - H3T11ph, and $L$ - H2AT121ph in metaphase II. Bars are $5 \mu \mathrm{m}$.

28, threonine 11 , as well as histone $\mathrm{H} 2 \mathrm{~A}$ at threonine 121 started in the (peri)centromeric regions at the beginning of meiotic prophase I and was detectable as dots at pachytene, randomly distributed over the nucleus (Fig. $5 A, D, G, J$ ). At metaphase I, the phosphorylation of histone $\mathrm{H} 3$ at serines 10 and 28 , as well as threonine 11 was spread throughout chromosomes (Fig. 5B,E,H). Te intensity of H3T11ph signals was considerably higher in centromeric regions (Fig. $5 H$ ). In rye and maize, an analogous increase in serine 10 and 28 histone H3 phosphorylation signals has been reported in the (peri)centromeric regions of metaphase I chromosomes (Gernand et al. 2003, Zhang et al. 2005). The phosphorylation of histone H2AT121 in the first meiotic division did not spread over the entire chromosome length and was detectable only in the centromeric regions (Fig. 5K) at metaphase I like in the case of maize meiotic cells (Dong and Han 2012).

In tobacco, signals of histone $\mathrm{H} 3$ phosphorylation at serines 10 and 28, and threonine 11 appeared at the centromeric regions at the very beginning of meiotic prophase I (Fig. 5A,D, $G$ ) and then could be detected along the entire chromosome length during metaphase I, like it was observed in wheat, rye and maize (Manzanero et al. 2000, Zhang et al. 2005). In contrast to that, signals of H2AT121ph during meiosis were detected only in the (peri)centromeric regions (Fig. $5 C, F, L$ ) of tobacco. At metaphase II, all H3 phospho-specific antibodies, except for H3T11ph, showed signals only in pericentromeric 


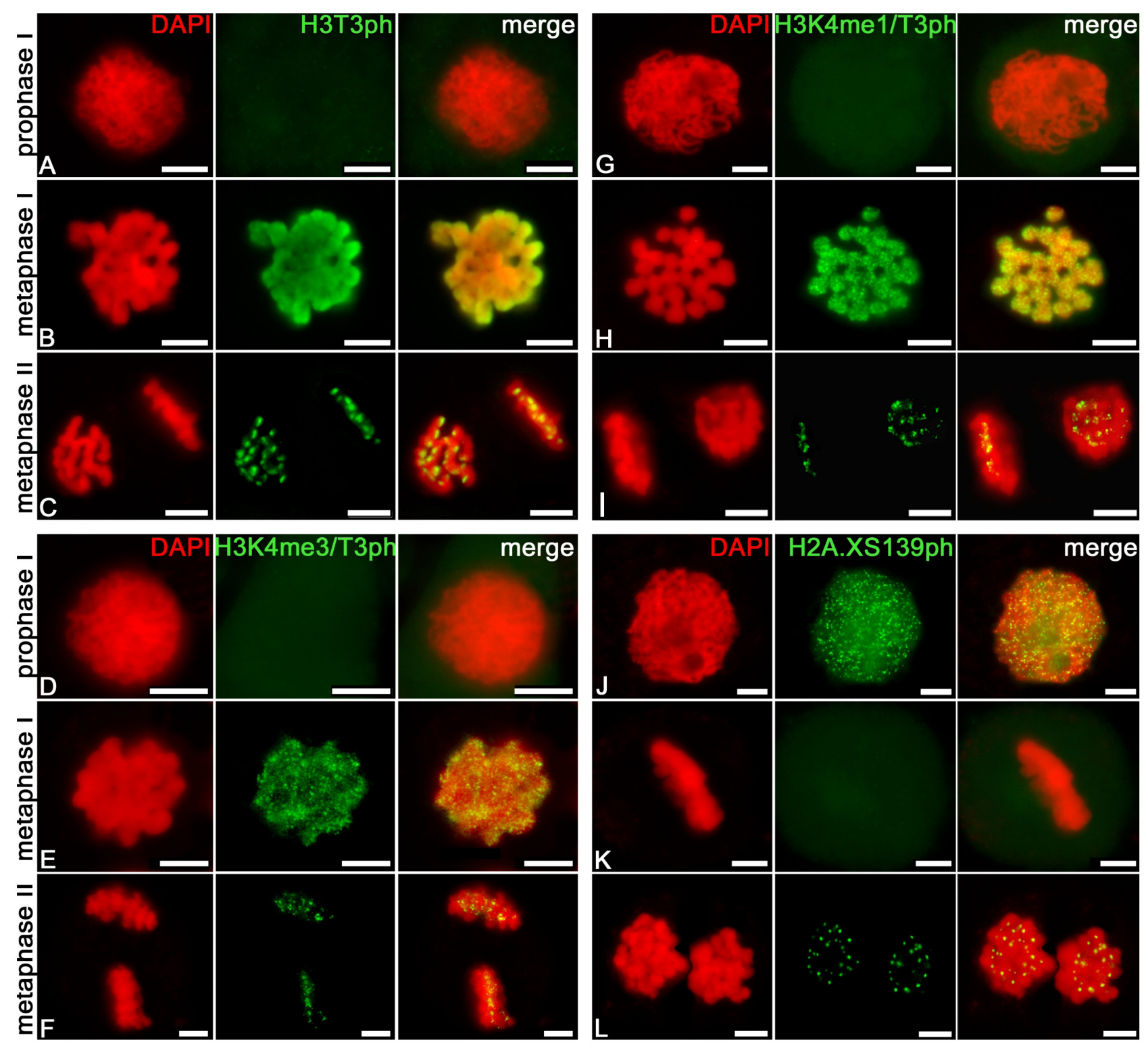

Fig. 6. The distribution of histones H3T3ph, H3K4me1/H3T3ph, H3K4me3/H3T3ph, and H2A.XS139ph during microsporogenesis of tobacco: $A$ - H3T3ph, $D$ - H3K4me3/H3T3ph, $G$ - H3K4me1/H3T3ph, and $J$ - H2A.XS139ph in prophase I (zygotene-pachytene); $B$ H3T3ph, $E$ - H3K4me3/H3T3ph, $H$ - H3K4me1/H3T3ph, and $K$ - H2A.XS139ph in metaphase I; $C$ - H3T3ph, $F$ - H3K4me3/H3T3ph, $I$ - H3K4me1/H3T3ph, and $L$ - H2A.XS139ph in metaphase II. Bars are $5 \mu \mathrm{m}$.

regions. Anti-H3T11ph showed signals over the entire length of chromosomes, and these signals were stronger at centromeric regions. Histone H3T3ph signals were absent at prophase I (Fig. 6A), however, this modification was spread over the entire chromosomes at metaphase I (Fig. 6b). In the second division, this histone mark is detectable only in centromeric regions (Fig. 6C). Antibodies recognizing the combination of phosphorylated threonine 3 and methylated lysine 4 detected patterns for these types of histone H3 modifications were similar in meiosis (Fig. 6D-I). The cell cycle dependent phosphorylation of histone $\mathrm{H} 3$ at threonine coincided with the euchromatin marks (H3K4me1,3), being uniformly spread over chromosomes in metaphase I
(Fig. $6 B, E, H$ ) and detectable only in chromosome (peri) centromeric regions in the second division (Fig. 6C,F,I). These results suggest that the (peri)centromeric regions of tobacco chromosomes, like (peri)centromeres of other eukaryotic chromosomes (Bergmann et al. 2011, Wu et al. 2011), also contain euchromatin.

Double immunostaining with H3K27me2 (a typical heterochromatin mark) and H3S10ph (a centromeric mark) antibodies demonstrated that these modifications did not co-localise (Fig. 4). In zygotene, the signals were localised to the opposite poles of the nucleus (Fig. 4A). In pachytene, clustering signals disappeared, and the signals were uniformly distributed throughout the nucleus 
(Fig. 4B). In metaphase I, the phosphorylation of serine 10 was spread over the entire surface of chromosomes (Fig.4C). However, H3S10ph signals in the second meiotic division again localised to the (peri)centromeric regions of chromosomes and did not co-localise with the H3K27me2 signal (Fig. 4D). The same dynamics of $\mathrm{H} 3 \mathrm{~S} 10$ phosphorylation was found in other plant species (Manzanero et al. 2000).

Thus double detection of H3S10ph and H3K27me2 in tobacco microsporocytes demonstrates that they clustered at opposite poles of the nucleus in the zygotene, which is characteristic of centromeric and telomeric chromosome regions (Wen et al. 2012, Zhang et al. 2013).

The histone H2A.X phosphorylated at serine 139 $(\gamma \mathrm{H} 2 \mathrm{~A} . \mathrm{X})$ is a marker for DNA double-strand break repairs (Rogakou et al. 1999, Xiao et al. 2009). In meiotic prophase I (zygotene-pachytene), anti-H2A. $\mathrm{XS139ph}$ detected numerous small signals dispersed throughout the nucleus in tobacco microsporocytes (Fig. $6 J$ ). The emergence of DNA double-strand breaks are most likely due to recombination processes taking place at this meiotic stage (Chicheportiche et al. 2007). These signals disappeared at the completion of prophase I and were undetectable in metaphase I (Fig. 6K). Such dot-like signals are also observed during the meiotic pachytene stage of rice ( $\mathrm{Ji}$ et al. 2016). In somatic cells (in interphase and mitosis) of $A$. thaliana (Amiard et al. 2010, Charbonnel et al. 2010) and Allium cepa (Zabka et al. 2014) phospho-H2A.X signals can be detected only after treatment with DNA damaging agents. At the same time, in somatic cells of Raphanus sativa, Vicia faba, and Allium porum, some phospho-H2A.X signals are detected even without induction of DNA damage (Rybaczek and Maszewski 2007). Signals of H2A.XS139ph in tobacco again appeared in a small number during the second meiotic division (Fig. 6L). Presumably, the reappearance of these signals was associated with DNA damage during the second meiotic division. A similar increase of phosphorylation of H2A.X in meiosis II at S139 was observed in meiotic cells of grasshopper (Cabrero et al. 2007). However, the role of this modification in the second phase of meiosis is unknown and may differ from DNA double-strand breaks repair.

In conclusion, histones and their posttranslational modification are conserved among eukaryotic species, but the comparison of the subnuclear and chromosomal distribution of methylated, phosphorylated, and acetylated histones revealed differences in their distribution patterns between species and between similar types of modifications in the same organism. The distribution of the main histone modification types in tobacco was similar to those described for other plant species but with some specific features. It was shown that hypermethylated heterochromatin blocks were located only in the part of tobacco chromosomes. Four euchromatin acetylation marks H3K9/14/18 and 27ac showed uniform distribution over the entire chromosome length, and only two of them, H3K18 and 27ac, formed hyperacetylation regions associated with the nucleolus and obviously involved in its functioning. Analysis of euchromatin mark distributions also revealed the presence of euchromatin in tobacco (peri)centromeres. Unusual phospho-H2A.X signals were observed in tobacco meiosis II.

\section{References}

Aliyeva-Schnorr, L., Beier S., Karafiatova, M., Schmutzer, T., Scholz, U., Dolezel, J., Stein, N., Houben A.: Cytogenetic mapping with centromeric bacterial artificial chromosomes contigs shows that this recombination-poor region comprises more than half of barley chromosome 3H. - Plant J. 84: 385394, 2015.

Amiard, S., Charbonnel, C., Allain, E., Depeiges, A., White, C.I., Gallego, M.E.: Distinct roles of the ATR kinase and the Mre11-Rad50-Nbs1 complex in the maintenance of chromosomal stability in Arabidopsis. - Plant Cell 22: 30203033, 2010.

An, G.: High efficiency transformation of cultured tobacco cells. - Plant Physiol. 79: 568-570, 1985.

Belyaev, N.D., Houben, A., Baranczewski, P., Schubert, I.: The acetylation patterns of histones $\mathrm{H} 3$ and $\mathrm{H} 4$ along Vicia faba chromosomes are different. - Chromosome Res. 6: 59-63, 1998.

Bergmann, J.H., Rodriguez, M.G., Martins, N.M.C., Kimura, H., Kelly, D.A., Masumoto, H., Larionov, V., Jansen, L.E.T., Earnshaw, W.C.: Epigenetic engineering shows $\mathrm{H} 3 \mathrm{~K} 4 \mathrm{me} 2$ is required for HJURP targeting and CENP-A assembly on a synthetic human kinetochore. - EMBO J. 30: 328-340, 2011.

Cabrero, J., Teruel, M., Carmona, F.D., Camacho, J.P.M.: Histone $\mathrm{H} 2 \mathrm{AX}$ phosphorylation is associated with most meiotic events in grasshopper. - Cytogen. Genome Res. 116: 311-315, 2007.

Carchilan, M., Delgado, M., Ribeiro, T., Costa-Nunes, P., Caperta, A., Morais-Cecilio, L., Jones, R.N., Viegas, W., Houben, A.: Transcriptionally active heterochromatin in rye B chromosomes. - Plant Cell 19: 1738-1749, 2007.

Charbonnel, C., Gallego, M.E., White, C.I. Xrcc1-dependent and $\mathrm{Ku}$-dependent DNA double-strand break repair kinetics in Arabidopsis plants. - Plant J. 64: 280-290, 2010.

Chicheportiche, A., Bernardino-Sgherri, J., De Massy, B., Dutrillaux, B.: Characterization of Spo11-dependent and independent phospho-H2AX loci during meiotic prophase I in the male mouse. - J. cell. Sci. 120: 1733-1742, 2007.

Dong, Q.H., Han, F.P.: Phosphorylation of histone H2A is associated with centromere function and maintenance in meiosis. - Plant J. 71: 800-809, 2012.

Fuchs, J., Demidov, D., Houben, A., Schubert, I.: Chromosomal histone modification patterns - from conservation to diversity. - Trends Plant Sci. 11: 199-208, 2006.

Fuchs, J., Schubert, I.: Chromosomal distribution and functional interpretation of epigenetic histone marks in plants. - In: Bass, W.H., Birchler, A.J. (ed): Plant Cytogenetics: Genome Structure and Chromosome Function. Pp. 231-253. Springer, New York 2012.

Gernand, D., Demidov, D., Houben, A.: The temporal and spatial pattern of histone $\mathrm{H} 3$ phosphorylation at serine 28 and serine 10 is similar in plants but differs between mono- and polycentric chromosomes. - Cytogenet. Genome Res. 101: 172-176, 2003.

Goodspeed, T.H.: The genus Nicotiana. - J. amer. pharmacol. Assoc. 45: 193, 1956.

Houben, A., Belyaev, N.D., Turner, B.M., Schubert, I.: Differential immunostaining of plant chromosomes by antibodies recognizing acetylated histone $\mathrm{H} 4$ variants. Chromosome Res. 4: 191-194, 1996. 
Houben, A., Demidov, D., Caperta, A.D., Karimi, R., Agueci, F., Vlasenko, L.: Phosphorylation of histone H3 in plants a dynamic affair. - Biochim. biophys. Acta 1769: 308-315, 2007.

Houben, A., Demidov, D., Karimi-Ashtiyani, R.: Epigenetic control of cell division. Epigenetic memory and control in plants. - In: Grafi, G., Ohad, N. (ed): Signaling and Communication in Plants. Pp. 155-175. Springer, Berlin Heidelberg 2013.

Houben, A., Demidov, D., Rutten, T., Scheidtmann, K.H.: Novel phosphorylation of histone $\mathrm{H} 3$ at threonine 11 that temporally correlates with condensation of mitotic and meiotic chromosomes in plant cells. - Cytogenet. Genome Res. 109: 148-155, 2005.

Jasencakova, Z., Meister, A., Schubert, I.: Chromatin organization and its relation to replication and histone acetylation during the cell cycle in barley. - Chromosoma 110: 83-92, 2001.

Ji, J., Tang, D., Shen, Y., Xue, Z., Wang, H., Shi, W., Zhang, C., Du, G., Li, Y., Cheng, Z.: P31comet, a member of the synaptonemal complex, participates in meiotic DSB formation in rice. - Proc. nat. Acad. Sci. USA. 113: 10577-10582, 2016.

Kaszas, E., Cande, W.Z.: Phosphorylation of histone H3 is correlated with changes in the maintenance of sister chromatid cohesion during meiosis in maize, rather than the condensation of the chromatin. - J. cell. Sci. 113: 3217-3226, 2000.

Kawashima, S.A., Yamagishi, Y., Honda, T., Ishiguro, K., Watanabe, Y.: Phosphorylation of H2A by Bub1 prevents chromosomal instability through localizing shugoshin. Science 327: 172-177, 2010.

Kenton, A., Parokonny, A.S., Gleba, Y.Y., Bennett, M.D.: Characterization of the Nicotiana tabacum L. genome by molecular cytogenetics. - Mol. gen. Genet. 240: 159-169, 1993.

Koukalova, B., Moraes, A.P., Renny-Byfield, S., Matyasek, R., Leitch, A.R., Kovarik, A.: Fall and rise of satellite repeats in allopolyploids of Nicotiana over ca. 5 million years. - New Phytol. 186: 148-160, 2010.

Kouzarides, T.: Chromatin modifications and their function. Cell 128: 693-705, 2007.

Lim, K.Y., Matyasek, R., Kovarik, A., Leitch, A.R.: Genome evolution in allotetraploid Nicotiana. - Biol. J. Linnean Soc. 82: 599-606, 2004.

Lim, K.Y., Matyasek, R., Lichtenstein, C.P., Leitch, A.R.: Molecular cytogenetic analyses and phylogenetic studies in the Nicotiana section Tomentosae. - Chromosoma 109: 245258, 2000.

Manzanero, S., Arana, P., Puertas, M.J., Houben, A.: The chromosomal distribution of phosphorylated histone H3 differs between plants and animals at meiosis. - Chromosoma 109: 308-317, 2000.

Murad, L., Lim, K.Y., Christopodulou, V., Matyasek, R., Lichtenstein, C.P., Kovarik, A., Leitch, A.R.: The origin of tobacco's $\mathrm{T}$ genome is traced to a particular lineage within Nicotiana tomentosiformis (Solanaceae). - Amer. J. Bot. 89: 921-928, 2002

Nakayama, J., Rice, J.C., Strahl, B.D., Allis, C.D., Grewal, S.I.: Role of histone $\mathrm{H} 3$ lysine 9 methylation in epigenetic control of heterochromatin assembly. - Science 292: 110-113, 2001.

Oliver, C., Pradillo, M., Corredor, E., Cunado, N.: The dynamics of histone $\mathrm{H} 3$ modifications is species-specific in plant meiosis. - Planta 238: 23-33, 2013.

Parokonny, A., Kenton, A.: Comparative physical mapping and evolution of the Nicotiana tabacum L. karyotype. - In: Brandham, P.E., Bennett, M.D. (ed.): Kew Chromosome Conference IV. Pp. 301-320. Royal Botanic Gardens, Kew 1995.

Ren, N., Timko, M.P.: AFLP analysis of genetic polymorphism and evolutionary relationships among cultivated and wild Nicotiana species. - Genome 44: 559-571, 2001.

Rogakou, E.P., Boon, C., Redon, C., Bonner, W.M.: Megabase chromatin domains involved in DNA double-strand breaks in vivo. - J. cell. Biol. 146: 905-916, 1999.

Rougeulle, C., Chaumeil, J., Sarma, K., Allis, C.D., Reinberg, D., Avner, P., Heard, E.: Differential histone H3 Lys-9 and Lys-27 methylation profiles on the X chromosome. - Mol. cell. Biol. 24: 5475-5484, 2004

Rybaczek, D., Maszewski, J.: Phosphorylation of H2AX histones in response to double-strand breaks and induction of premature chromatin condensation in hydroxyurea-treated root meristem cells of Raphanus sativus, Vicia faba, and Allium porrum. Protoplasma 230: 31-39, 2007.

Salina, E.A., Sergeeva, E.M., Adonina, I.G., Shcherban, A.B., Afonnikov, D.A., Belcram, H., Huneau, C., Chalhoub, B.: Isolation and sequence analysis of the wheat $\mathrm{B}$ genome subtelomeric DNA. - BMC Genomics 10: 414, 2009.

Sharma, S.K., Yamamoto, M., Mukai, Y.: Immuno-cytogenetic manifestation of epigenetic chromatin modification marks in plants. - Planta 241: 291-301, 2015.

Shibata, F., Nagaki, K., Yokota, E., Murata, M.: Tobacco karyotyping by accurate centromere identification and novel repetitive DNA localization. - Chromosome Res. 21: 375-381, 2013.

Vershinin, A.V., Schwarzacher, T., Heslop-Harrison, J.S.: The large-scale genomic organization of repetitive DNA families at the telomeres of rye chromosomes. - Plant Cell 7: 1823$1833,1995$.

Wen, R.Y., Moore, G., Shaw, P.J.: Centromeres cluster de novo at the beginning of meiosis in Brachypodium distachyon. - PLoS ONE 7: e44681, 2012.

Wu, Y., Kikuchi, S., Yan, H., Zhang, W., Rosenbaum, H., Iniguez, A.L., Jiang, J.: Euchromatic subdomains in rice centromeres are associated with genes and transcription. - Plant Cell 23: 4054-4064, 2011.

Xiao, A., Li, H.T., Shechter, D., Ahn, S.H., Fabrizio, L.A., Erdjument-Bromage, H., Ishibe-Murakami, S., Wang, B., Tempst, P., Hofmann, K., Patel, D.J., Elledge, S.J., Allis, C.D.: WSTF regulates the H2A.X DNA damage response via a novel tyrosine kinase activity. - Nature 457: 57-62, 2009.

Zabka, A., Polit, J.T., Bernasinska, J., Maszewski, J.: DNA topoisomerase II-dependent control of the cell cycle progression in root meristems of Allium cepa. - Cell Biol. Intern. 38: 355-367, 2014.

Zhang, J., Pawlowski, W.P., Han, F.P.: Centromere pairing in early meiotic prophase requires active centromeres and precedes installation of the synaptonemal complex in maize. Plant Cell 25: 3900-3909, 2013.

Zhang, X., Li, X., Marshall, J.B., Zhong, C.X., Dawe, R.K.: Phosphoserines on maize centromeric histone $\mathrm{H} 3$ and histone H3 demarcate the centromere and pericentromere during chromosome segregation. - Plant Cell 17: 572-583, 2005. 